\title{
Upper Extremity Tendon Transfers: A Brief Review of History, Common Applications, and Technical Tips
}

\author{
Jason Gardenier ${ }^{1}$ Rohit Garg ${ }^{2}$ Chaitanya Mudgal ${ }^{2}$ \\ ${ }^{1}$ Division of Plastic Surgery, Harvard Combined Plastic Surgery \\ Residency Program, Massachusetts General Hospital, Fruit Street, \\ Boston MA, United States \\ ${ }^{2}$ Orthopaedic Surgery, Harvard Medical School, Hand Surgery \\ Service, Massachusetts General Hospital, Fruit Street, Boston MA, \\ United States \\ Indian J Plast Surg:2020;53:177-190
}

\author{
Address for correspondence Chaitanya Mudgal, MD, MS (Orth.), \\ M.Ch (Orth.), Harvard Medical School, Hand Surgery Service, \\ Massachusetts General Hospital, Fruit Street, Boston, MA 02114, \\ United States (e-mail: cmudgal@partners.org).
}

\begin{abstract}
Keywords

- Extensor tendons

- Flexor tendons

- Tendon reconstruction

- Tendon transfer

Background Tendon transfer in the upper extremity represents a powerful tool in the armamentarium of a reconstructive surgeon in the setting of irreparable nerve injury or the anatomic loss of key portions of the muscle-tendon unit. The concept uses the redundancy/expendability of tendons by utilizing a nonessential tendon to restore the function of a lost or nonfunctional muscle-tendon unit of the upper extremity. This article does not aim to perform a comprehensive review of tendon transfers. Instead it is meant to familiarize the reader with salient historical features, common applications in the upper limb, and provide the reader with some technical tips, which may facilitate a successful tendon transfer.

Learning Objectives (1) Familiarize the reader with some aspects of tendon transfer history. (2) Identify principles of tendon transfers. (3) Identify important preoperative considerations. (4) Understand the physiology of the muscle-tendon unit and the Blix curve. (5) Identify strategies for setting tension during a tendon transfer and rehabilitation strategies.

Design This study was designed to review the relevant current literature and provide an expert opinion.

Conclusions Tendon transfers have evolved from polio to tetraplegia to war and represent an extremely powerful technique to correct neurologic and musculotendinous deficits in a variety of patients affected by trauma, peripheral nerve palsies, cerebral palsy, stroke, and inflammatory arthritis. In the contemporary setting, these very same principles have also been very successfully applied to vascularized composite allotransplantation in the upper limb.
\end{abstract}

\section{Introduction}

Tendon transfer in the upper extremity is a transformative tool which can restore lost functions suffered as a result of central or peripheral neurologic injury or traumatic loss of a muscle-tendon unit (MTU). A tendon transfer makes use of the redundancy and expendability in muscles of the upper extremity to separate a nonessential MTU from its anatomic insertion and reroute it to perform a function for which it was not originally designed. This tool allows surgeons to use functional, but nonessential, MTUs to help perform other functions which are deficient due to tendon rupture, muscle death, irreparable peripheral nerve injury, or central nervous system dysfunction.
License terms

()(1) $\odot \circledast$ 


\section{History}

The conceptual foundation of using a dispensable muscle to replace the function of a paralyzed or lost muscle lies in the polio epidemics in Europe in the 19th century which left many children paralyzed at the inception of early modern surgery. The father of this technique is generally considered to be Carl Nicoladoni who first described its use in Innsbruck, Austria, using the peroneus longus tendon to treat paralysis from polio in $1882 .{ }^{1}$ This approach gained in popularity largely due to further advances by Tomasz Drobnik, ${ }^{2}$ who described it in Konigsberg, Germany, to treat foot drop and was the first to use the technique in the upper extremity, describing its use in radial nerve palsy. Steindler in 1918 described the importance of preserving the blood supply to the transferred tendon to restore elbow flexion $^{3}$ which allowed for further improvement in outcomes. Incorporating lessons learned from the world wars, especially World War II, in 1960 Bunnell and Boyes further emphasized the atraumatic technique which is essential to preserve the integrity of the transferred tendon. ${ }^{4}$ These authors are generally considered to be the fathers of the contemporary state of the art of tendon transfers in the upper extremity. In the modern era, tendon transfers are widely used for a variety of deficits including replacement of a ruptured or avulsed tendon/muscle, ${ }^{5}$ peripheral nerve injuries with no hope of improvement, ${ }^{6}$ central neurological deficits such as spinal cord injury or cerebral palsy, and more recently in vascularized composite allotransplantation (VCA) in the case of hand transplantation. ${ }^{7.8}$ These techniques are sometimes accompanied by additional treatments including nerve transfer, dynamic splinting, tendon grafting, tenodesis, arthrodesis, and functional free muscle transfer.

\section{Key Principles of Tendon Transfer}

In 1916, Biesalski and Mayer described five main principles of tendon transplantation. ${ }^{9}$

- Restoration of tendon-sheath relationship.

- Direct the tending through tissues which allow tendon gliding.

- Imitate normal insertion.

- Establish normal tension.

- Preserve an effective line of tension.

\section{Preoperative Considerations for the Soft Tissues and Skeleton}

To accomplish the goals mentioned above, it is important to ensure the following prior to embarking upon surgery.

- Neurologic recovery has plateaued.

- Tissues have achieved equilibrium (meaning that inflammation and edema have subsided to minimize scarring/ tethering).

- There is a stable and healthy soft tissue bed with a stable skeleton.
- Joints affected by the transfer are supple/mobile. Any skeletal injury or fracture should ideally be completely stably fixed or healed.

Technical tip: A silicone Hunter rod may be used to preserve or create a tendon path if the proposed path of the tendon transfer is deemed unsuitable. ${ }^{10}$ In such a situation, staged surgery is prudent. In the first stage, the Hunter rod is placed along the course of the proposed tendon transfer. In the second stage, which is usually performed 3 to 4 months after the first stage, the Hunter rod is removed and the path left behind by the rod is now utilized to facilitate passage of the tendon transfer. If a Hunter rod is not available, a sterile $1 / 4$ inch Penrose drain or sterile intravenous tubing may also be utilized as alternate techniques to prepare the path for the future tendon transfer.

Timing of the tendon transfer can be dependent upon numerous variables and oftentimes surgery may need to be deferred until a suitable time. In such situations, it is vitally important that the joints that will be affected by the tendon transfer or crossed by the tendon transfer be maintained as supple as possible. An early referral and assessment by a hand therapist is crucial to ensure suppleness of the joints with passive mobilization techniques. Should joints have become stiff or develop flexion deformities, the use of serial casting and wedging as well as joint mobilization techniques is really important to ensure adequate suppleness of the joints in preparation for the future tendon transfer. Suppleness of joint movement which allows complete or near complete passive range of motion of joints is mandatory and critical prior to considering the transfer.

\section{Donor Selection}

The method for donor selection forms a key part in decision making for a tendon transfer. It necessitates a careful and thorough assessment of what muscle units are working, their strength, and the desired function after the transfer. The donor and recipient should be matched in terms of strength, amplitude, excursion, direction of pull, and function. Excursion of the tendons in the wrist and hand may best be thought of via the 3-5-7 rule. ${ }^{7}$ Wrist flexors/extensors provide $33 \mathrm{~mm}$ of excursion, while finger extensors provide $50 \mathrm{~mm}$, and finger flexors provide $70 \mathrm{~mm} .{ }^{11}$ Tenodesis effect in the setting of a mobile wrist can augment finger extension by up to $2.5 \mathrm{~cm}$ when the wrist is flexed. ${ }^{12}$ It is essential to select a donor of adequate strength to replace the desired function, while also remembering that the strength of the muscle is proportional to the cross-sectional area of the muscle belly. Accordingly, the strongest forearm muscles are the brachioradialis (BR) and the flexor carpi ulnaris (FCU), with a relative strength (RS) of 2, followed by the flexor carpi radialis (FCR), wrist extensors, and finger flexors, with a RS of 1 ; finger extensors with a RS of 0.5 , and finally abductor pollicis longus (APL), extensor pollicis longus (EPL), extensor pollicis brevis (EPB), and palmaris longus (PL), with a RS of 0.1. ${ }^{13,14}$

The donor should be expendable and it is recommended to use redundant muscles such as using one of the three wrist 
extensors-extensor carpi ulnaris (ECU), extensor carpi radialis longus (ECRL), or extensor carpi radialis brevis (ECRB). Ideally there should be synergistic properties of functional muscle groups, such as utilizing a wrist flexor to augment finger extension.

\section{Additional Preoperative Considerations}

Additional factors to consider prior to tendon transfer include the following. (1) Managing the patient's goals and expectations and making sure that they are realistic and aligned with the goals of surgery. (2) Assess whether the neurologic deficit is evolving and if so, delaying the transfer until recovery has plateaued is prudent. (3) The emotional and social factors impacting the patient, living situation, ability of caregivers to support the patient in the postoperative recovery phase including transporting the patient to their rehabilitation appointments. (4) The patient's functional requirements, both vocational as well as avocational, must be recognized. (5) The patient's visual deficits if any must be identified early, as these can adversely impact ability to participate in postoperative rehabilitation. (6) The sensibility of the hand which is extremely critical in recovery and may dictate choice of treatment if the hand is completely insensate.

\section{Muscle-Tendon Unit Physiology/ Biomechanics}

Understanding the physiology and biomechanics of the MTU is key to a successful outcome. At the most basic level, this means maximizing the overlap of actin and myosin filaments in the resting state to allow for maximum possible tendon excursion ( - Fig. 1). This is conceptualized by the Blix curve (-Fig. 2), which describes the relationship between the resting length of a muscle fiber and its ability to generate tension in response to nerve stimulation or passive stretch. ${ }^{15}$ In short this curve shows that as muscle length increases beyond the resting length, the ability of the muscle to generate tension via contraction drops rapidly. However, beyond a certain length the tension does rise simply due to the passive stretch of the muscle. This was demonstrated experimentally by Lieber et al in 1992 by using laser diffraction to measure sarcomere length in muscles, ${ }^{16}$ and this work was later advanced by further experiments by Takahashi et al. ${ }^{17}$ The resting length is the position from which the muscle generates its greatest force in response to motor stimulation and contraction of the sarcomere. Viscoelastic force of muscle, which is the combined resistance to stretch produced by muscle, fascia, and connective tissue, increases with stretch but also adversely affects the force generated by the muscle.

Adjusting the resting tension during tendon transfer is crucial and is referred to as "tensioning." If a tendon transfer is too loose, there is too much actin-myosin overlap at rest and contraction will not translate into adequate tendon excursion. However, there are also are adverse consequences to overtightening a tendon transfer as there is then not enough actin-myosin overlap, since the sarcomere is lengthened. Recent experiments have shown that overtightening a tendon transfer can reduce the force of muscle to $28 \%$ of its maximal force. ${ }^{18}$

In 2007, Takahashi et al demonstrated that a surgeon could measure sarcomere length using laser diffraction during in vivo tendon transfer in rabbit experiments, but the clinical utility in humans is not known. ${ }^{17}$ Takahashi et al also showed that after a tight tendon transfer, muscles will naturally stretch over a period of weeks with increased sarcomere number, and then increased tendon length in response to excess tendon tensioning, but it is unclear whether function improves. ${ }^{19}$ If the tendon transfer is overtightened by overstretching of the MTU, the contractile force decreases; however, the tension in muscle via passive stretch increases ( - Fig. 2). In this overtightened state, the tendon transfer works more via a tenodesis effect rather than by generating a contractile force. For example, if an FCR to EDC transfer in a radial nerve palsy is overtightened, the ability to perform active finger extension will be limited and will mostly be obtained in a position of wrist flexion via the tenodesis effect.

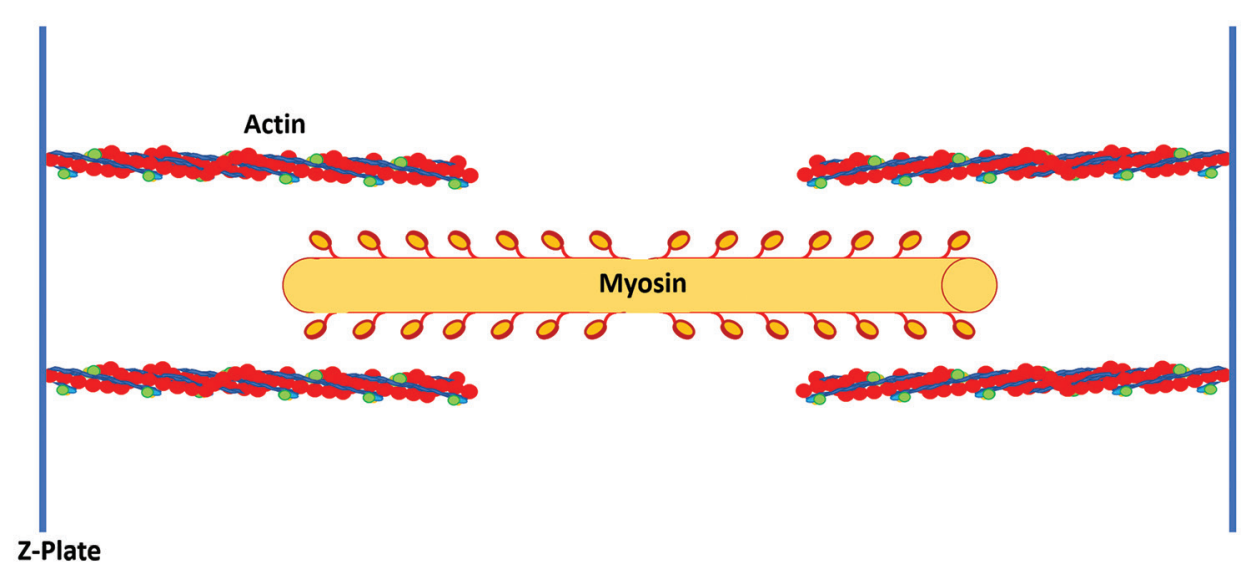

Fig. 1 The relationship of actin and myosin molecules within a sarcomere determines the level of force generated by the muscle and the amount of excursion of the tendon. 


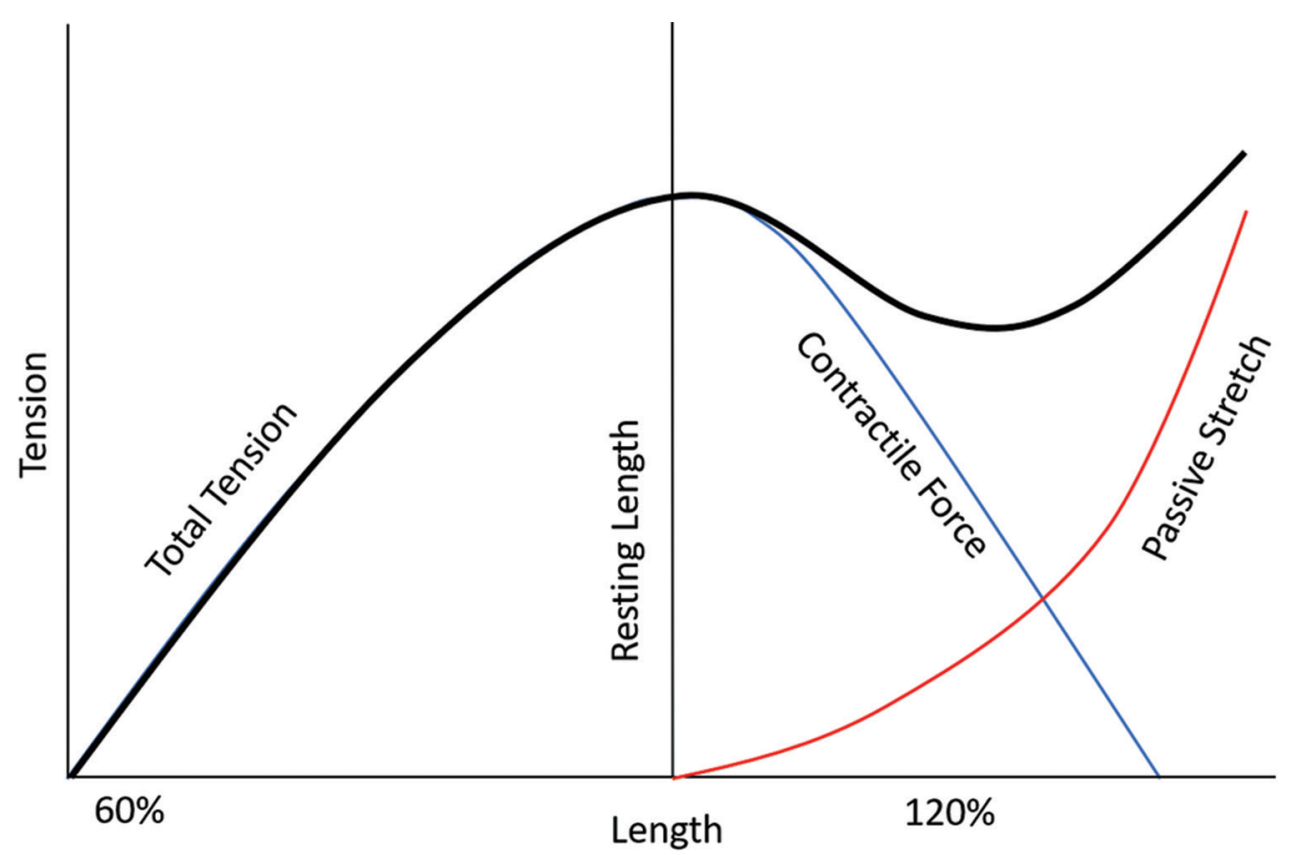

Fig. 2 The Blix curve was described by Magnus Blix in the late $1800 \mathrm{~s}^{40}$ and is the summation of two tension-length curves-one for active contraction and one for elastic recoil when plotted against the length, demonstrating greatest force generation when stimulated, at the resting length of the muscle-tendon unit.

\section{Current Applications}

Current applications include the replacement of ruptured tendons, treatment of peripheral nerve palsies, cerebral palsy, or other conditions such as VCA. However, peripheral nerve palsies are the most common indication for upper extremity tendon transfers. Functional recovery after peripheral nerve injuries is variable and can be incomplete. If functional recovery after peripheral nerve injuries is not sufficient for adequate function, tendon transfers currently remain the treatment of choice in most parts of the world.

\section{Technical Considerations}

Several technical considerations are important to optimize function and minimize complications after upper extremity tendon transfers. These include the type of tendon coaptation, when to consider arthrodesis, and how to manage immobilization and therapy postoperatively. The Pulvertaft weave was described in 1954 and consists of interweaving the donor and recipient tendons three to four times. ${ }^{20}$ After the first weave has been taken, a suture is placed and the tension of the transfer is assessed. The remaining weaves serve to augment the strength of the repair. While this technique has been remarkably effective, durable, and studied extensively, it can be time consuming and create a bulky repair. More recent biomechanical studies have suggested that the sideto-side coaptation technique is at least as strong if not stronger and may reduce the bulk of the repair to some extent..$^{21,22}$ Another important consideration is early mobilization, and some authors state that mobilization must begin within 24 to 48 hours to minimize risk of adhesions and joint stiffness. ${ }^{23-25}$ Traditionally though, most surgeons have relied on at least a brief period of immobilization of 2 to 3 weeks after the procedure, before commencing rehabilitation. While the literature certainly supports the role of early rehabilitation, this must be carefully weighed against the patient's ability to comprehend and be compliant with these rehabilitation programs. Finally, it is important to consider situations, such as cerebral palsy, or stroke, or traumatic brain injury, when arthrodesis may provide more stability and a superior outcome to tendon transfer in some circumstances.

\section{Radial Nerve Injury}

Radial nerve injury results in inability to extend the wrist, fingers, and thumb, and this is the most common indication for upper extremity tendon transfers. ${ }^{7}$ High radial nerve injuries occur proximal to the elbow and result in all of the above deficits by loss of innervation to the BR, ECRL, ECRB, extensor digitorum communis (EDC), extensor digiti quinti (EDQ), ECU, APL, EPB, EPL, and extensor indicis proprius (EIP). Low radial nerve injuries occur below the elbow and mainly affect the posterior interosseous nerve. In this injury wrist extension is relatively preserved due to proximal branches to the ECRL. Commonly performed tendon transfers include pronator teres (PT)-ECRB transfer for wrist extension. Thumb extension is accomplished by transferring PL or the ring finger flexor digitorum superficialis (FDS) to the EPL. Finger extension is usually restored by transferring FCR, FCU, or FDS to the EDC. Of these, the FDS provides the best tendon excursion, but sacrifices some of the synergy for digital flexion. We prefer to not use the FCU, as we consider it vital to effective ulnar deviation of the wrist in flexion, and its role in the dart thrower's motion of the wrist. Multiple tendon transfers for 

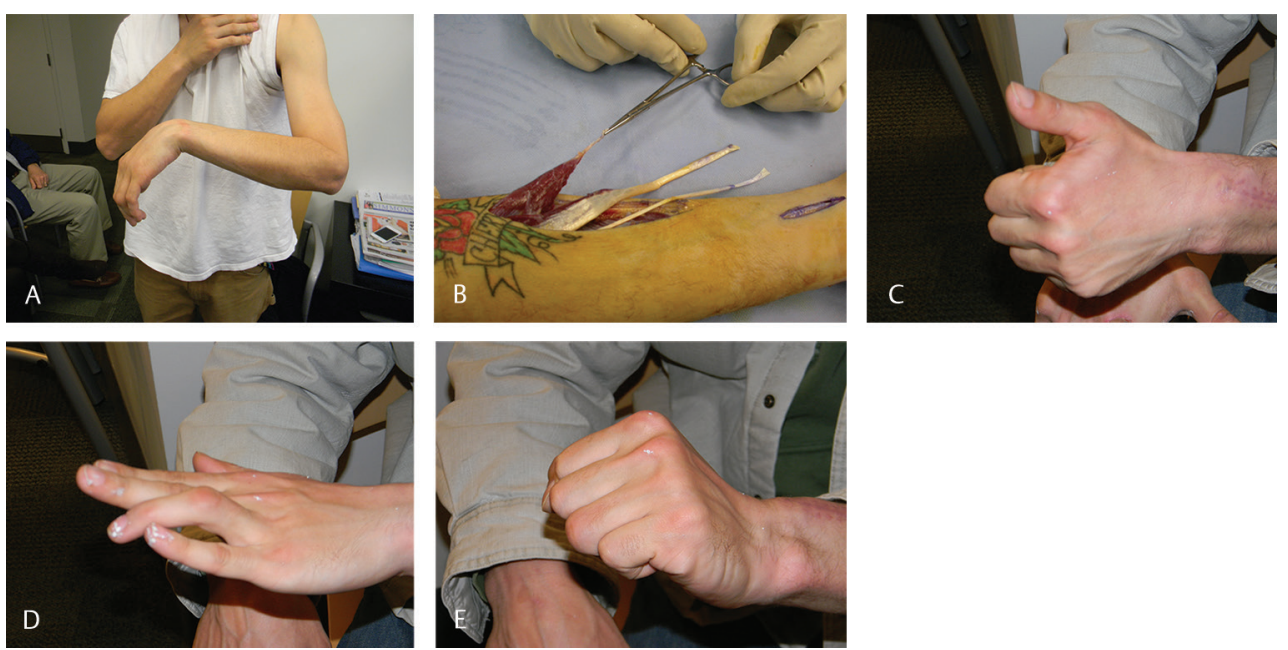

Fig. 3 (A) Clinical appearance in radial nerve palsy following a high-energy humeral shaft fracture and no clinical functional recovery after 12 months. (B) In this case PT (instrument) was transferred to ECRB, FCR (red arrow) was transferred to EDC, and PL (yellow arrow) was transferred to EPL. (C-E) Nine months postoperative follow-up shows excellent restoration of the wrist, thumb, and digital extension. The patient had returned to work and had recently sustained an unrelated traumatic boutonniere of the ring finger, which was also treated nonoperatively. For intraoperative tensioning of transfers, please see - Video 1. ECRB, extensor carpi radialis brevis; EDC, extensor digitorum communis; EPL, extensor pollicis longus; FCR, flexor carpi radialis; PL, palmaris longus; PT, pronator teres.

radial nerve palsy are shown in - Fig. 3, including PT-ECRB, FCR-EDC, and PL-flexor pollicis longus (FPL) ${ }^{26}$

Technical tip: The preferred technique utilized by the senior author (C.M.) is the Jones transfer. This consists of transferring the PT to the ECRB to restore wrist extension, the FCR to the EDC to restore finger extension, and the PL to the EPL to ensure thumb interphalangeal (IP) joint extension.

The PT has a short tendon and the length of this is often inadequate to effectively create a substantive Pulvertaft weave. Therefore, it is recommended that the tendon is lengthened by elevating it with a strip of periosteum to increase its effective length and make the Pulvertaft weave more substantive and stronger. However, in circumstances where this is also not adequate, the senior author (C.M.) chooses to augment this repair by one the of two techniques.

In the first technique the PT tendon is elongated by sewing a long strip of dorsal forearm fascia which is harvested while exposing the ECRB. This forearm fascia is sewn to the PT tendon to make it longer. This allows a very substantive Pulvertaft weave which is quite strong and four weaves can be achieved. Alternatively, either a strip of forearm fascia or a strip of the partial ECRL tendon can be harvested and sewn on either side of the Pulvertaft weave to the ECRB and the PT tendon respectively, to give an additional layer of strength to the transferred tendon. Use of the forearm fascia in this manner to augment a tendon repair is called fascial augmentation tenorrhaphy, and is a useful tool in augmenting tenuous tendon repairs or tendon transfers. ${ }^{26}$

Setting tension: The first step is to transfer the PT to the ECRB. This is done with the wrist maintained in 50 to 60 degrees of extension and some length may be gained in the PT by flexion of the elbow. Once the tension has been set, the wrist is then supported with a bump of towels to maintain it in its position. After the Pulvertaft weave is completed, it should be possible to let the wrist be lowered gently passively to just short of neutral.

The next step is to set the tension for the digital extensors. The FCR is transferred around the radial border. The digital extensors are sewn together to make one mass proximal to the extensor retinaculum and are not sectioned by the senior author. The FCR is transferred in such a way so that its angle of approach to the EDC is as close to a straight line of pull as possible. Tension is set so that, with the wrist in 30 to 40 degrees of extension, it should be possible to flex the metacarpophalangeal (MP) joints to approximately 50 to 60 degrees of flexion; in addition, lowering the wrist to neutral should allow the MP joints to extend fully.

The EPL is released completely from its third compartment so that it can be moved radially subcutaneously, and it is not sectioned. This allows the transfer to extend as well as abduct the thumb. In a similar way tension is set so that, after the PL is sewn into the EPL with the wrist in extension, it should be possible passively for the thumb tip to oppose to the tip of the small finger. As the wrist is brought down to neutral the thumb should automatically extend at both the MP and IP joints ( - Video 1).

\section{Video 1}

Intraoperative video demonstrating transfers utilized for radial nerve palsy and the methods for setting tension as well as augmenting the PT-ECRB transfer. ECRB, extensor carpi radialis brevis; PT, pronator teres. Online content including video sequences viewable at: https://www. thieme-connect. com/products/ejournals/html/ 10.1055/s-0040-1716456.

Rehabilitation: In the immediate postoperative setting, the patient's upper limb is immobilized in a splint with the 
elbow at 90 degrees of flexion, the wrist in maximal extension, the forearm in pronation, and the MP joints at neutral extension. The IP joints are left free and an active range of motion of the IP joints is encouraged. After suture removal is done in 12 to 14 days, the patient's upper limb is then placed in a long-arm cast in the position described above. The cast is discontinued 4 weeks postoperatively. The patient is then transitioned to an Orthoplast splint with the elbow free, the wrist in 30 degrees of extension, and MP joints at neutral. Retraining of the transfers under the supervision of a hand therapist is commenced at this time. Six weeks after surgery, the patient is transitioned to night splinting only. Eight to 10 weeks after surgery splinting is discontinued and strengthening is commenced. Unrestricted activity is usually allowed after 12 weeks.

Functional gains: It has been reported by Dabas et al that the PT to ECRB transfer good resulted in a $48 \%$ increase in grip strength and a $90 \%$ increase in strength of key pinch 6 months postoperatively. In addition, Skoll and colleagues found that transferring the FCR restored nearly $79 \%$ of the power grip compared with the unaffected side. ${ }^{27,28}$

\section{Ulnar Nerve Injury}

Ulnar nerve injuries can be more devastating than radial nerve injuries as the ulnar nerve innervates almost all the intrinsic muscles of the hand. Specifically, the ulnar nerve innervates FCU and part of the FDP along with the hypothenar muscles, the third and fourth lumbricals, all of the dorsal and palmar interossei, the adductor pollicis, and sometimes the flexor pollicis brevis. Complete ulnar nerve palsies result in clawing of the hand and loss of key pinch.

Loss of key pinch arises from paralysis of the adductor pollicis and first dorsal interosseous muscles. Clawing results from intrinsic muscle paralysis. In the normal hand, MP joint extension by the extrinsic extensors is balanced by MP joint flexion by the intrinsic flexors. However, in an ulnar palsy once the intrinsic flexors are dysfunctional, the extrinsic extensors are able to extend the MP joints without opposition, which in turn increases the tension of the long (extrinsic) flexor tendons leading to flexion at the IP joints. This is made even more obvious because IP joint extension is lost due to intrinsic muscle dysfunction. This leads to the classic claw which affects the ulnar digits more.

In addition, patients also complain of asynchronous digital flexion and during the course of which, the normal cascade of flexion which starts at the MP joints and progresses to the IP joints is lost. Instead, the fingers flex first at the IP joints and come into contact with the palm before MP joint flexion is completed. This results in a rolling motion of the digits. Therefore, in the act of grasping an object, the object is actively pushed away by the fingertips, as opposed to grasping it. This is illustrated in -Videos $\mathbf{2}$ and $\mathbf{3}$ (courtesy of Hill Hastings II, MD), showing the difference in grasping a round object in an intrinsic minus hand as well as the difference in flexion in normal and intrinsic minus hands. It must be remembered that the degree of clawing is much worse in the low ulnar nerve palsy as compared with a high ulnar nerve palsy. This is because in a high ulnar nerve palsy the profundus tendons to the ring and small fingers are also paralyzed. This is known as the "ulnar paradox."

\section{Video 2}

Asynchronous flexion in an ulnar nerve palsy. Notice the inability to grasp the object as it slides away from the hand as it closes. (These images are provided courtesy of Hill Hastings II, MD.) Online content including video sequences viewable at: https://www. thieme-connect. com/products/ejournals/html/ 10.1055/s-0040-1716456.

\section{Video 3}

Comparative depiction of synchronous and asynchronous digital flexion, which highlights clawing and lack of intrinsic function. (These images are provided courtesy of Hill Hastings II, MD.) Online content including video sequences viewable at: https://www. thieme-connect. com/products/ejournals/html/ 10.1055/s-0040-1716456.

Functional restoration in ulnar nerve dysfunction consists of restoration of profundus function to the ring and small fingers, restoration of synchronous finger flexion, restoration of key pinch, and correction of clawing.

In a high ulnar nerve palsy, small-finger and ring-finger FDP function may be restored by suturing these FDP tendons to the long-finger FDP to allow en-masse flexion of these three digits.

Technical tip: The index finger profundus tendon is not included in the suture, so as to preserve its independent function.

Key pinch can be restored by transferring ECRB or FDS to adductor pollicis. The ECRB is divided at its insertion and withdrawn proximal to the extensor retinaculum. It is then passed superficial to the extensor retinaculum and lengthened with the help of a tendon graft (either the PL or a toe extensor). A generous opening is created in the interosseous space between the second and third metacarpals. Using the third metacarpal as a pulley, this tendon is now routed deep to the other flexors and superficial to the adductor pollicis to be attached to the insertion of the adductor pollicis or to the proximal phalanx of the thumb.

The ring or long-finger FDS tendons may also be transferred to the adductor pollicis, though with a less effective line of pull and some sacrifice of grip strength.

Technical tip: In high ulnar nerve palsy, the FDS of the ring finger should not be used for pinch restoration, as that would lead to the sacrifice of the lone functioning long flexor in the ring finger.

Clawing can be corrected via a variety of techniques. Prior to deciding which technique to utilize in the correction of clawing, the surgeon must perform the Bouvier test.

Technical tip: The Bouvier test differentiates between simple clawing and complex clawing. The MP joint of the 
affected digits are placed into flexion and the patient is then asked to extend the IP joints. If the patient is able to do so, the test is considered to be positive and the clawing is then considered simple clawing. If the patient is unable to extend the IP joints, then the test is considered to be negative and the clawing is considered a complex clawing ( - Video 4; courtesy of Hill Hastings II, MD).

\section{Video 4}

The Bouvier test is demonstrated. Notice the improvement in IP extension with blocking of MP extension. An "anticlaw" splint placed in this manner can often be a reasonable form of management for patients with simple clawing, who are not suitable or willing for surgery. (These images are provided courtesy of Hill Hastings II, MD.) Online content including video sequences viewable at: https://www. thieme-connect.com/products/ ejournals/html/ 10.1055/s-0040-1716456.

Patients who have simple clawing may benefit from a static procedure. The idea behind such a procedure is to simply place the MP joints into flexion so that the patient may be able to extend the IP joints. These procedures do not address asynchronous finger flexion and also do not contribute to an increase in grip strength. Therefore, they may be considered as "static" procedures. A distally based flap of the volar plate of the MP joint as described by Zancolli is advanced proximally and reattached to the metacarpal neck either with the help of sutures or suture anchors. Another simple procedure which was described by Bunnell is a partial release of the A1 and A2 pulleys, which in effect causes bowstringing of the flexor tendons promoting MP joint flexion and limiting their hyperextension. ${ }^{29,30}$

Alternatively, the FDS may also be utilized to create a static flexion moment arm as described by Zancolli, known commonly as the "lasso" procedure. In low ulnar nerve palsies, the FDS to the ring and small fingers is divided at its insertion or just proximal to it. It is then passed either distal to the A1 pulley or distal to both A1 and A2 pulleys and then brought back and sewn to itself while maintaining the MP joints in the intrinsic plus position of approximately 60 to 70

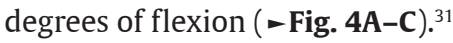

Technical tip: In some patients, the FDS to the small finger may be absent or very gracile. In these situations, the ring-finger FDS may be split into two slips and one slip is rerouted to the small finger to perform the lasso. In high ulnar nerve palsies, the FDS of the ring and small fingers cannot be sacrificed as the patients do not have a functioning FDP. Instead of the ring- and small-finger FDS, the middle-finger FDS is utilized to perform the lasso. The method of harvesting is similar, and it is divided into two slips, each of which is then rerouted to the ring and small fingers to perform the lasso.

Dynamic tendon transfers are utilized in complex clawing. Not only do they provide dynamic correction by flexing the MP joint and extending the IP joint, but they also can provide increased grip strength. Dynamic tendon transfers consist of either utilizing the FDS or wrist extensor with tendon grafts. The modified Stiles-Bunnell tendon transfer for clawing involves sacrificing the long-finger FDS and splitting it in four slips. Each of these slips is then passed along the path of the lumbricals, volar to the deep transverse metacarpal ligament, to insert along the lateral bands or into the proximal phalanges. ${ }^{32,33}$

Technical tip: In patients who have ligamentous laxity or pre-existing hyperextension of the proximal IP (PIP) joint, procedures that involve sacrificing the FDS and its subsequent insertion into the lateral band may not be suitable as they may lead to hyperextension at the PIP joint. In such patients with low ulnar nerve palsies, the middle-finger FDS may be utilized to perform this transfer. In this situation it is divided into two slips, one each for the ring and small fingers. An alternative technique is to insert the slip of the FDS into the proximal phalanx and not the lateral band to avoid hyperextension at the PIP joint ( $\mathbf{- F i g . 5 A - C}$ ).

Another dynamic tendon transfer consists of using the ECRL. ${ }^{33}$ This needs to be augmented with tendon grafts. The ECRL tendon is divided in its insertion. It is then passed superficial to the extensor retinaculum. Tendon grafts are harvested and sewn to the ECRL tendon. Through generous openings which are made in the second and fourth intermetacarpal spaces, these tendon grafts are passed from dorsal to volar along the course of the lumbricals to be inserted onto the lateral bands in the digits.

Technical tip: The tendon grafts are passed first and sewn on the radial side of the index finger and on the ulnar side of the middle, ring, and small fingers. These grafts are then
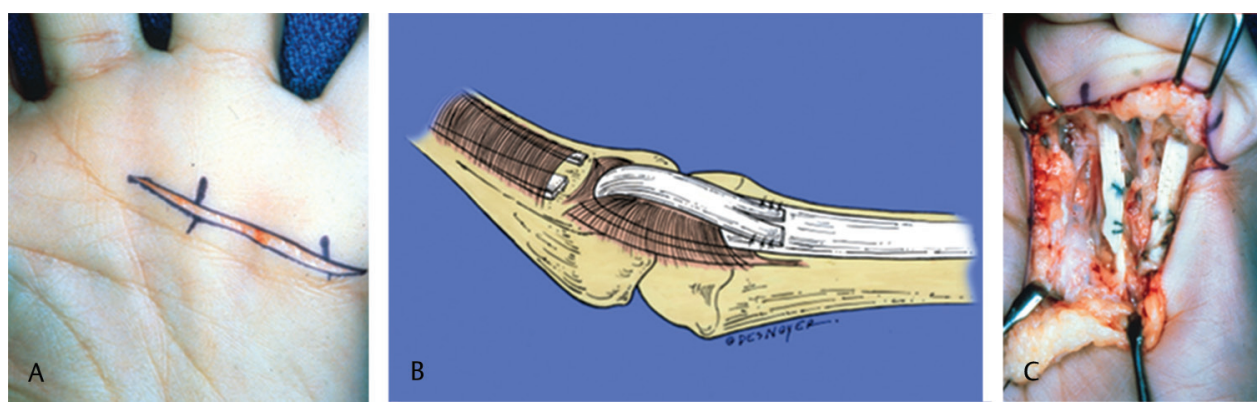

Fig. 4 (A) A transverse incision is centered over the A1 pulley. (B) Method of detaching the FDS and passing it around the A1 pulley before sewing it to itself. (C) The completed "lasso" procedure as described by Zancolli. (These images are provided courtesy of Hill Hastings II, MD.) FDS, flexor digitorum superficialis. 

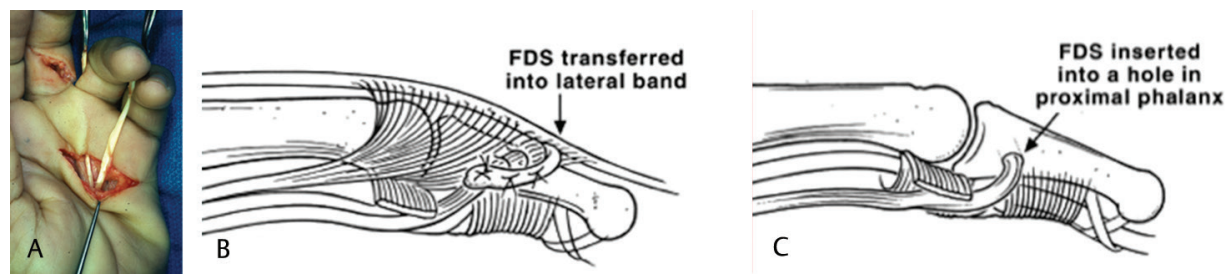

Fig. 5 (A) A modified Stiles-Bunnell transfer using the FDS of the middle finger. The tendon is split into slips that are passed along the lumbrical canal, volar to the deep transverse metacarpal ligament, and inserted either into the (B) radial lateral band or (C) proximal phalanx. (These images are provided courtesy of Hill Hastings II, MD.) FDS, flexor digitorum superficialis.
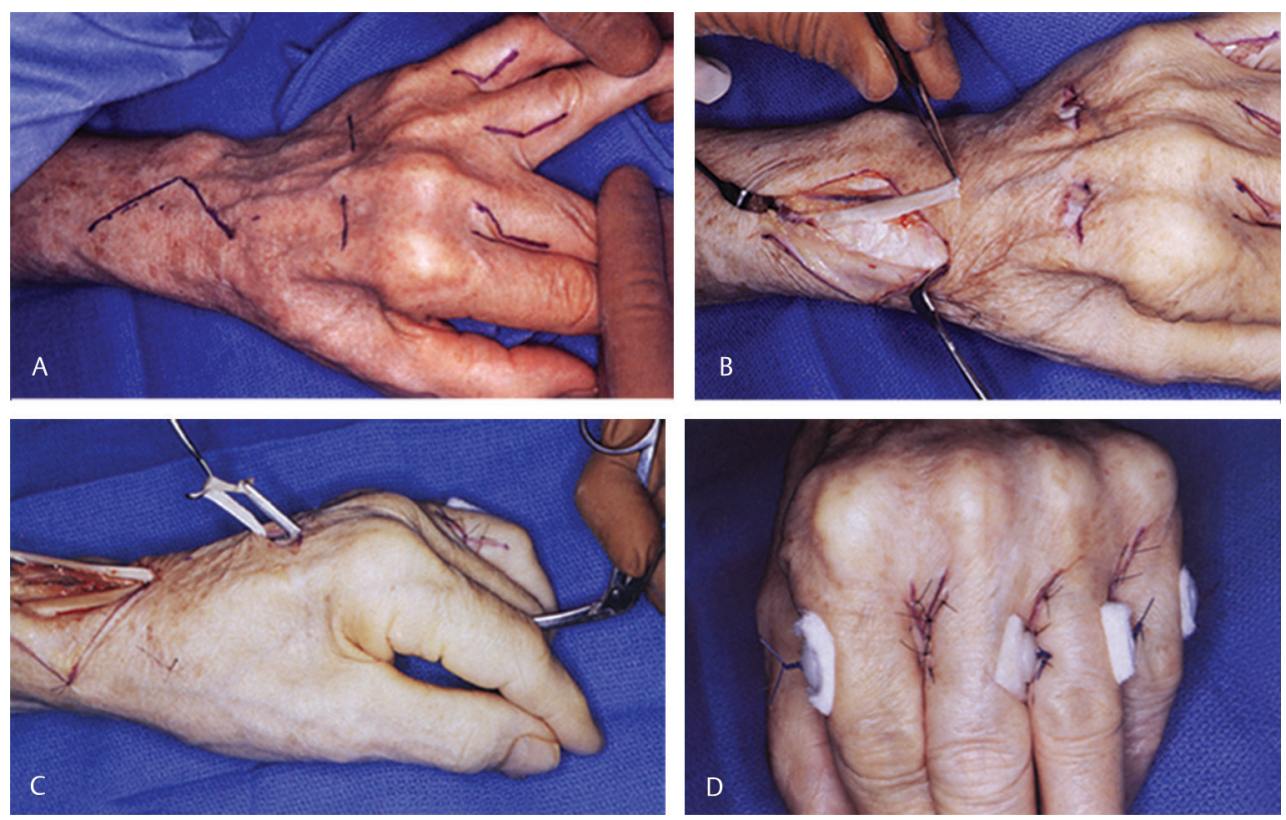

Fig. 6 (A) Incisions utilized in performing a transfer of the ECRL with tendon grafts for dynamic correction of clawing. The transfers are attached on the radial lateral bands of the middle, ring, and small fingers and the ulnar lateral band of the index finger. Alternatively, they are inserted into the proximal phalanx. (B) The ECRL is divided at its insertion and then passed superficial to the extensor retinaculum. (C) The grafts are sutured distally and then passed to emerge into the dorsal intermetacarpal wounds, where they are tensioned as described in the text. (D) The completed appearance. In this case the grafts were inserted into the proximal phalanx and pullout sutures placed in the grafts were tied over a button. (These images are provided courtesy of Hill Hastings II, MD.) ECRL, extensor carpi radialis longus.

retrieved dorsally in the second and fourth intermetacarpal spaces. The digital wounds are closed. The tendon grafts are sewn to each other before sewing them altogether to the ECRL ( - Fig. 6A-D).

Setting tension: Tension is set, so as to allow complete digital extension with the wrist extended. With mild wrist flexion, the MP joints should flex fully while still allowing complete IP extension.

Rehabilitation: In patients who undergo a Zancolli lasso, postoperative splinting consists of a dorsal blocking splint with the wrist at neutral, the MP joints flexed 60 degrees, and the IP joints in complete extension. They are transitioned to an Orthoplast splint of a similar nature on the fifth postoperative day. Unlimited flexion is allowed. Attempted MP joint extension is allowed only after 6 weeks. Strengthening is also commenced at 6 weeks. Night splinting is continued for a further 6 weeks.

In patients who undergo transfer of the ECRL, postoperative immobilization consists of a plaster splint with the wrist extended 30 to 40 degrees and the fingers in intrinsic plus position. Two weeks after surgery, they are transitioned to an Orthoplast splint with the wrist in 30 degrees of extension and the fingers in intrinsic plus position. Gentle active flexion is commenced at this time. Six weeks after surgery, patients are transitioned to an MP extension blocking splint only. Strengthening is commenced at this time.

Functional gains: Restoration of key pinch appears to confer a uniform functional improvement, irrespective of the techniques used. In one study after FDS transfer in 22 patients, grip strength was noted to improve from 30 to $71 \%$ of the contralateral side. ${ }^{34}$ In patients who underwent the ECRB transfer, Smith noted doubling of pinch strength from their preoperative status, in almost all the patients in his study. ${ }^{35}$ Most patients with a simple claw will have a successful correction of the claw after a Zancolli lasso. However, a lasso procedure does not lead to strength augmentation. Correction of asynchronous finger flexion has been demonstrated to occur in $93 \%$ of patients after a Stiles-Bunnell transfer. Grip strength improvement is best noted after procedures using either the ECRL or ECRB as motors. $^{36-38}$ 


\section{Median Nerve Injury}

Complete high median nerve palsies result in a loss of the palmar sensation, including the thumb, index finger, long finger, and half of the ring finger, as well as paralysis of FDS, FCR, PL, PT, FPL, PQ the FDS of the index and long fingers, and all of the thenar muscles (the abductor pollicis brevis [APB], opponens pollicis, and the superficial head of the FPB. Because the sensory loss on the palmar aspect of the radial digits can directly impact hand function, some surgeons may have reservations about performing tendon transfers in isolation. Instead they may combine these with nerve transfers to restore some sensation to the radial side of the hand.

Boatwright and Kiebzak studied the contribution of the median nerve in abduction strength of the thumb. They found that thenar muscle contribution to abduction strength varies from 70 to $74 \%{ }^{39}$ Therefore, the loss of thumb opposition is the most critical, as this deficit robs patients of a key human technical ability to abduct the thumb. Restoration of thumb opposition is achieved with an opponensplasty.

In low median nerve injuries, restoration of opposition can be achieved by using the ring-finger FDS, ${ }^{40,41}$ or the PL, as these muscles retain their innervation in this situation. ${ }^{42}$

Technical tip: When performing an opponensplasty, ideally the tendons that are transferred to the thumb approach the thumb from the base of the hypothenar eminence, ensuring the line of pull is in the direction of the pisiform, so as to mimic true opposition rather than function as mere abductors. In low median nerve injuries or advanced carpal tunnel syndrome with thenar wasting, especially in the older population, the Camitz opponensplasty is utilized. The PL is used in this transfer. However, by itself the PL does not have length to reach the insertion of the abductor on the base of the proximal phalanx (P1) of the thumb. Therefore, the PL with an extension of palmar fascia (usually $\sim 2 \mathrm{~cm}$ in width) is elevated carefully off the transverse carpal ligament and the extension of the palmar fascia may be taken as far distal as the distal palmar crease. Meticulous dissection is necessary to ensure continuity of the palmar fascia with the PL. In addition, great care is taken to avoid injury to the branches of the median nerve as well as the superficial palmar arch (-Fig. 7A-C). Meticulous hemostasis is critical while harvesting the palmar fascia to avoid a postoperative hematoma from the extensive subdermal plexus in the palm. This transfer behaves more as an abductorplasty rather than a true opponensplasty, since the line of pull is more in the direction of the forearm rather than the pisiform.

An alternative method is to utilize the FDS of the ring finger. The FDS is harvested in the distal palm and withdrawn at the wrist. The FCU is exposed and a strip of FCU is then harvested but left attached to the pisiform. It is then sewn to itself to create a loop. The FDS tendon is now passed through this loop and subcutaneously directed across the palm and attached to the base of the proximal phalanx of the thumb. This creates a line of pull in the direction of the pisiform in an effort to mimic a true opposition motion (-Fig. 8A-E and - Video 5). It is the senior author's (C.M.) preference to utilize the Camitz opponensplasty in patients who are older and have low median nerve dysfunction. Alternatively, the FDS to the ring finger is utilized. Should either of these not be available, the EIP is utilized by rerouting it around the ulnar border of the hand.

\section{Video 5}

Active opposition after opponensplasty using an FDS transfer through a loop of FCU. The line of pull of the transfer is toward the pisiform, making for a better opponensplasty rather than abductorplasty. FCU, flexor carpi ulnaris; FDS, FDS, flexor digitorum superficialis. Online content including video sequences viewable at: https://www. thieme-connect.com/products/ejournals/ html/ 10.1055/s-0040-1716456.

In high median nerve injuries, in performing an opponensplasty, the EIP or the abductor digiti minimi, EDQ, ECU, or ECRL may be used. ${ }^{43,44}$ In addition, in high median nerve injuries, the FDP function to the index and long fingers can be restored by transferring the BR, ECRL, or ECU to the FDP of the index and long fingers. Alternatively, the FDP to the index and long fingers may be sewn to the FDP of the ring and small fingers to create a "mass effect" digital flexion. BR to FPL remains a common tendon transfer to restore thumb flexion, and this is most commonly performed concomitantly with an ECRL to index and long FDP tendon transfer (- Fig. 9A-D).

Technical tip/setting tension: In the performance of a Camitz opponensplasty, the transfer is sewn to the base of P1 and the insertion of the APB with the thumb in maximal abduction as the wrist is placed in neutral on the operating
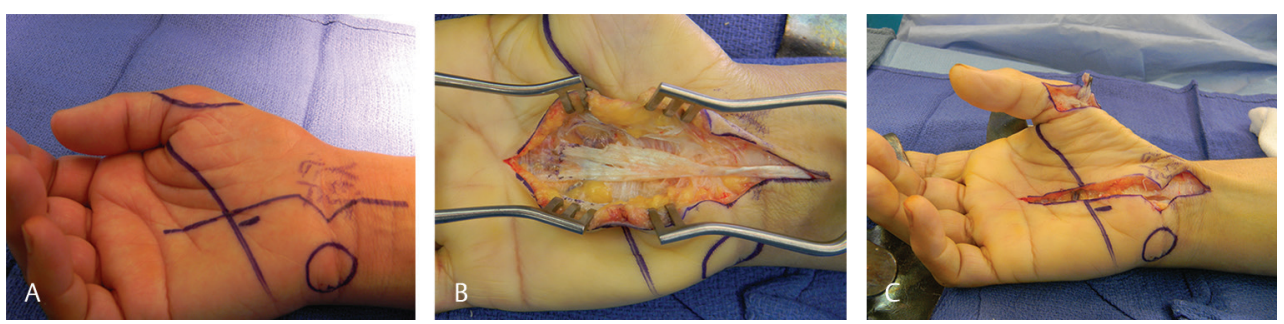

Fig. 7 Camitz opponensplasty in a patient with advanced carpal tunnel syndrome. (A) Incisions and marked thenar atrophy. (B) The palmaris longus is harvested with an extension of palmar fascia as shown, prior to distal division and elevation. (C) Position of thumb after transfer to the base of the proximal phalanx. Note the line of pull is directed more toward the forearm, making this more of an abductorplasty rather than a true opponensplasty. 

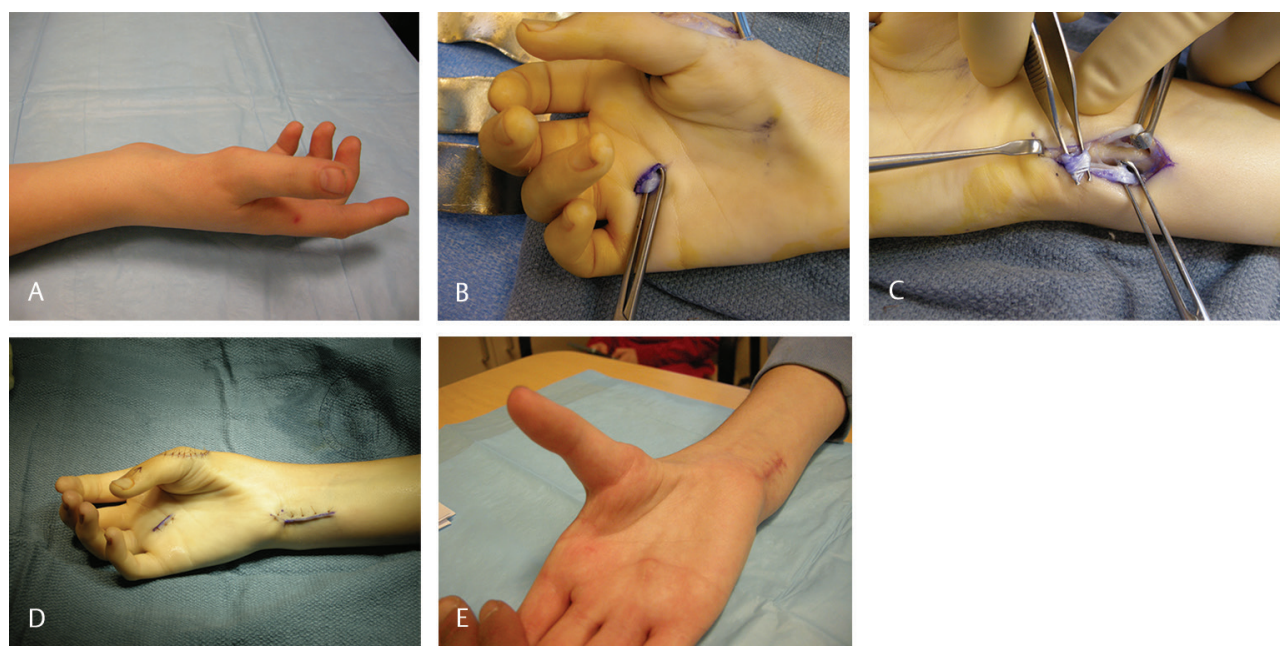

Fig. 8 (A) Opponensplasty in a patient with a Type 1 hypoplastic thumb and obvious thenar muscle absence. (B) The FDS tendon of the ring finger is isolated. (C) It is then rerouted through a loop formed with a distally based loop of half the FCU, and inserted on the APB to restore thumb opposition. (D) Incisions utilized and clinical appearance at the end of surgery. (E) Postoperative follow-up shows excellent thumb opposition. Active opposition may also be seen in -Video 5. APB, abductor pollicis brevis; FDS, flexor digitorum superficialis; FCU, flexor carpi ulnaris.
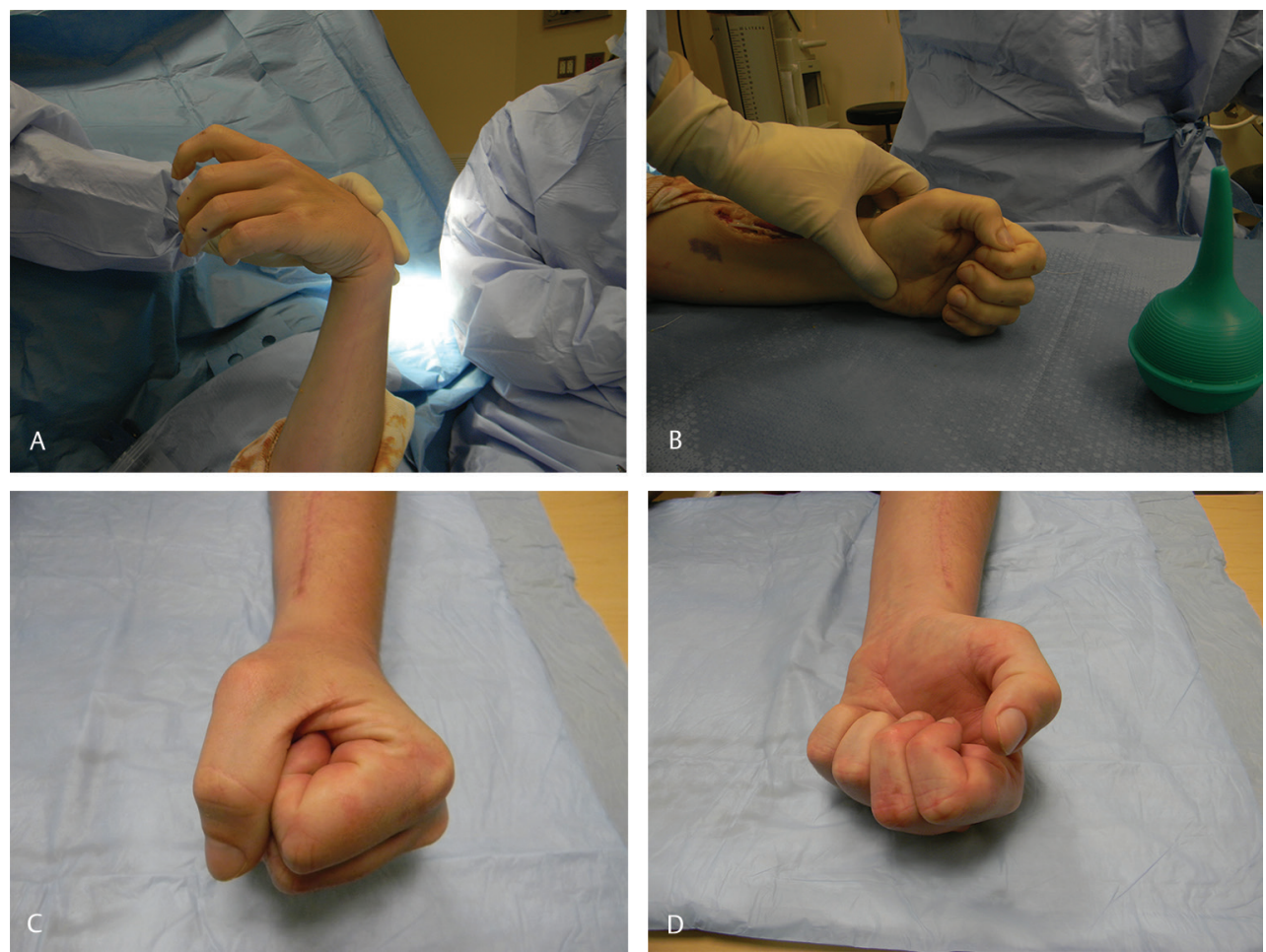

Fig. 9 (A) Setting tension in a patient undergoing ECRL to FDP of index and middle fingers in a patient with a high median nerve palsy, who recovered thenar function after nerve grafting but had weak flexion of index and middle fingers and no function in the FPL. With wrist flexion, the fingers extend in a natural cascade. (B) With the wrist extended, there is an excellent flexion cascade and thumb pinch to the index finger tip. (C, D) Postoperative function showing excellent restoration of finger and thumb flexion. ECRL, extensor carpi radialis longus; FDP, flexor digitorum profundus; FPL, flexor pollicis longus.

table. A similar strategy is also utilized in transferring the FDS of the ring finger to the base of proximal phalanx of the thumb.

In high median nerve palsy, the ECRL is transferred to the FDP of the index and middle fingers and the BR is transferred to the FPL. To restore opposition in this circumstance, it is the senior author's preference to utilize the EIP. The elbow is placed in 90 degrees of flexion and the ECRL is sewn into the FDP of the index and middle fingers such that, with the wrist extended 30 degrees, the profundii are tight enough that the fingertips reach about a centimeter away from the palmar skin. With the wrist flexed the finger should extend practically fully. In addition, as the elbow is extended, further augmentation of the tension in the transfer is noticeable. When transferring the BR, a similar methodology is utilized. The elbow is maintained in 90 degrees of flexion and tension 
is set in such a way that with the wrist at neutral and the elbow flexed, it is possible for the tip of the thumb to oppose the distal IP of the index finger to mimic generation of key pinch. This is further augmented as the wrist is extended and the elbow is extended, mimicking a very strong pinch ( - Fig. 9A-D). The EIP is harvested proximal to the extensor hood at the MP joint and retrieved at the wrist, proximal to the extensor retinaculum. It is then routed subcutaneously around the ulnar border to the base of $\mathrm{P} 1$ of the thumb. The thumb is placed in maximal abduction while setting tension. The sequence of transfers is: ECRL-EIP-BR.

Rehabilitation: Postoperatively the patient's upper limb is immobilized in a short arm thumb spica cast (with the IP joint free) for the Camitz and FDS ring-finger procedures, for a period of 3 to 4 weeks. Patients are then transitioned to an Orthoplast long opponens splint and the transfer is re-trained under the supervision of a hand therapist. Six weeks after surgery, the patient transitions to a night splint only while doing strengthening during the daytime. Nine to 10 weeks after surgery, splinting is discontinued.

In the case of a high median nerve palsy, a long arm thumb spica cast is used for 3 to 4 weeks, with the thumb and finger tips included. The rest of the rehabilitation is similar to that described above for a low median nerve palsy. In an effort to ascertain the integrity of the tendon transfer and assure its meticulous handling postoperatively, it is the practice of the senior author (C.M.) to apply all postoperative casts for tendon transfers, by himself.

Functional gains: When using the FDS to perform an opponensplasty, up to $40 \%$ of thenar strength can be restored. ${ }^{45}$ When using the EIP for opponensplasty, one study found good to excellent outcomes in $87 \%$ of patients. This study quantified a good result as one in which the patient was able to oppose the tip of the thumb to that of the index and middle fingers and an excellent result as one in which the patient could oppose the tip of the thumb to the ring or the small finger ${ }^{46}$ In patients who undergo a Camitz opponensplasty, Terrono and colleagues found a very high rate of patient satisfaction in $94 \%$ of patients. ${ }^{47}$

\section{Rheumatoid Arthritis and Traumatic Rupture}

Tendon transfers are also used to repair tendon ruptures associated with rheumatoid arthritis (RA) secondary to tenosynovitis, osteophyte, or spicule formation. In RA, the distal radio-ulnar joint (DRUJ) is affected by synovial hypertrophy and this in turn leads to DRUJ arthritis with bony spicule formation as well as dorsal subluxation of the ulnar head, called the "caput ulnae" syndrome. The combination of ulnar head subluxation, synovitis, and bony spicules can cause attritional rupture of digital extensor tendons, and the transfer of tendons for reconstruction usually necessitates resection of the ulnar head to prevent recurrent attritional rupture of the transferred tendon.

The most common tendon to rupture in RA is FPL, which is usually caused by bony spurs in the carpal tunnel, a condition commonly referred to as "Mannerfelt syndrome." ${ }^{48}$ This ruptured FPL can be reconstructed by transferring the
FDS of the middle or the long finger. ${ }^{49}$ Attritional rupture of extensor tendons, including EPL and finger extensors, also occurs. ${ }^{50}$ Finger extensor rupture in RA usually starts at the small finger, and may progress radially to affect the ring, long, and index fingers as well. When associated with subluxation of the ulnar head or DRUJ arthritis, this is referred to as the Vaughan-Jackson syndrome. ${ }^{51}$ These are usually reconstructed by transferring adjacent tendons, if possible (-Fig. 10A-D).

Technical tip: When assessing tendon ruptures in RA, it is critical to have a high index of suspicion for more ruptures than may be clinically obvious. Surgical planning should take into account the possibility of having to reconstruct more tendons than those obviously clinically ruptured.

If three extensors are involved, then if possible we use adjacent tendons for all of these. Alternatively, two tendons may be reconstructed with transfers using adjacent tendons and one tendon may also be reconstructed with a tendon graft, usually sourced from either the PL or a toe extensor. If all digital extensors are affected, then transferring either the FCR and/or FDS of the middle finger may be required. In most circumstances of tendon ruptures on the ulnar side of the wrist in RA, there are degenerative changes in the DRUJ. Hence, all tendon reconstructions are usually accompanied by excision of the distal ulna.

Setting tension: The tendon transfers are set with tension to allow nearly 50 to 60 degree MP flexion with the wrist extended to 30 degrees. With the wrist being brought back to neutral, the fingers should extend in a near-normal cascade at the MP joints.

Rehabilitation: When tendon reconstructions and transfers are performed for tendon ruptures in RA, the distal ulna is usually excised. If the distal ulna is stabilized with a tendon transfer, then the limb is immobilized postoperatively in supination in a long-arm cast with the elbow at 90 degrees of flexion. If the distal ulna does not require stabilization with a tendon transfer, the limb is then immobilized in a long-arm cast with the elbow at 90 degrees of flexion and the forearm at neutral rotation. The wrist is maintained in the cast in approximately 30 degrees of extension and the MP joints are maintained at neutral extension. The IP joints are left free. While in the cast, the patient is actively encouraged to move the IP joints as much as possible. Three weeks after surgery cast is discontinued, and the patient's upper limb is then immobilized in a forearm-based resting Orthoplast splint with the wrist in 30 degrees of extension and the MP joints at neutral. The IP joints are left free. Active and active assisted range of motion of the MP joints is commenced. Wrist range of motion is also commenced. Forearm rotation is also allowed. Six weeks after surgery, splinting is discarded and gentle progressive strengthening is commenced.

Isolated rupture of the EPL is also seen in patients with undisplaced fractures of the distal radius. The EPL is usually reconstructed using EIP. It is the practice of the senior author (C.M.) to use a "two-level" repair when transferring the EIP to EPL. This affords an extremely strong repair and allows commencement of early active rehabilitation within 

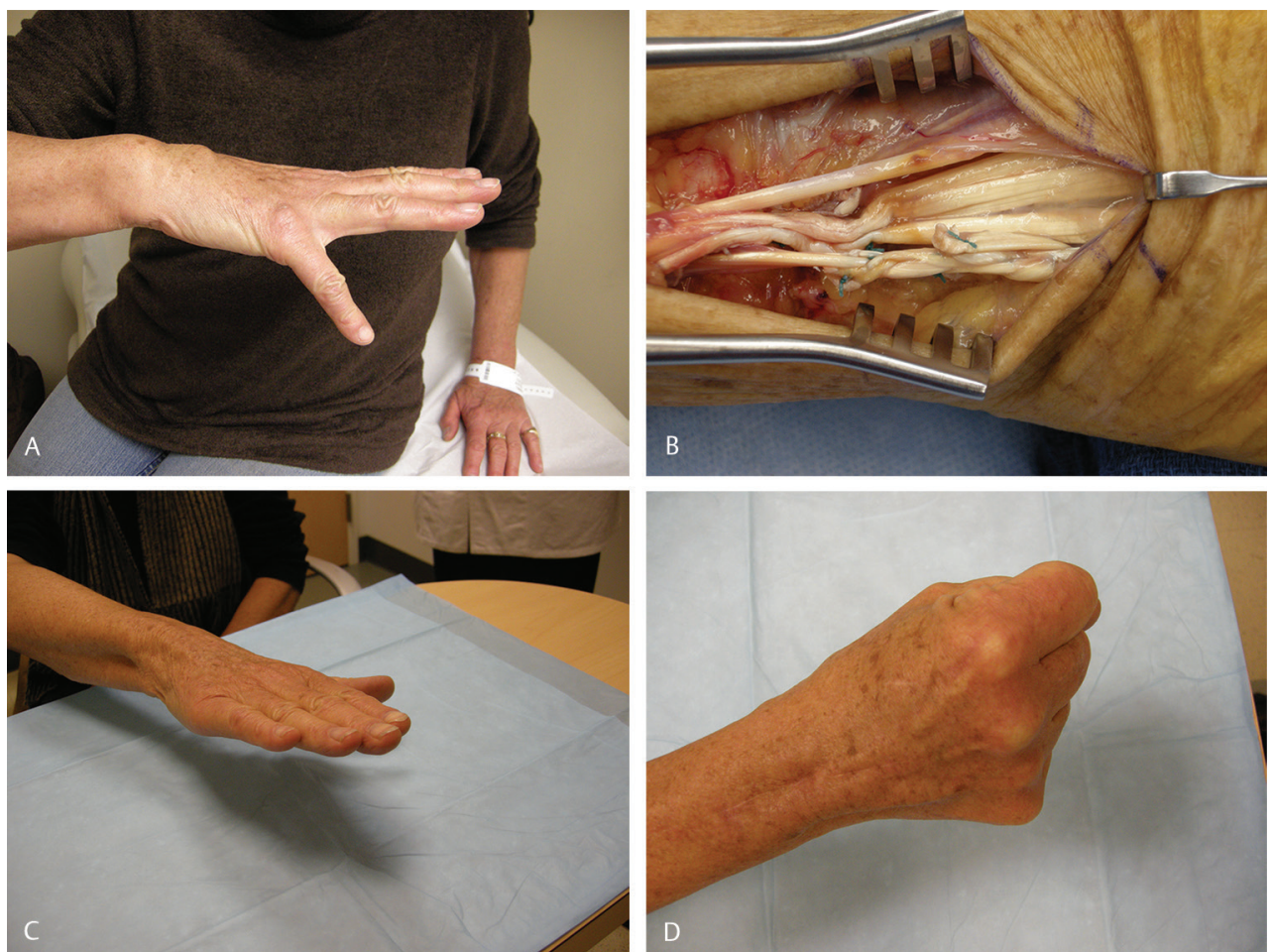

Fig. 10 (A) Clinical appearance in a patient with RA and extensor tendon ruptures to the small finger. (B) Intraoperatively, there was evidence of a near complete rupture of the extensor to the ring finger as well. Therefore, the EDC of the ring finger was reconstructed by transferring it to the intact EDC of the middle finger. The EIP was transferred to the EDC of the small finger. The distal ulna was excised. (C, D) Clinical appearance 12 weeks after surgery. EDC, extensor digitorum communis; EIP, extensor indicis proprius.
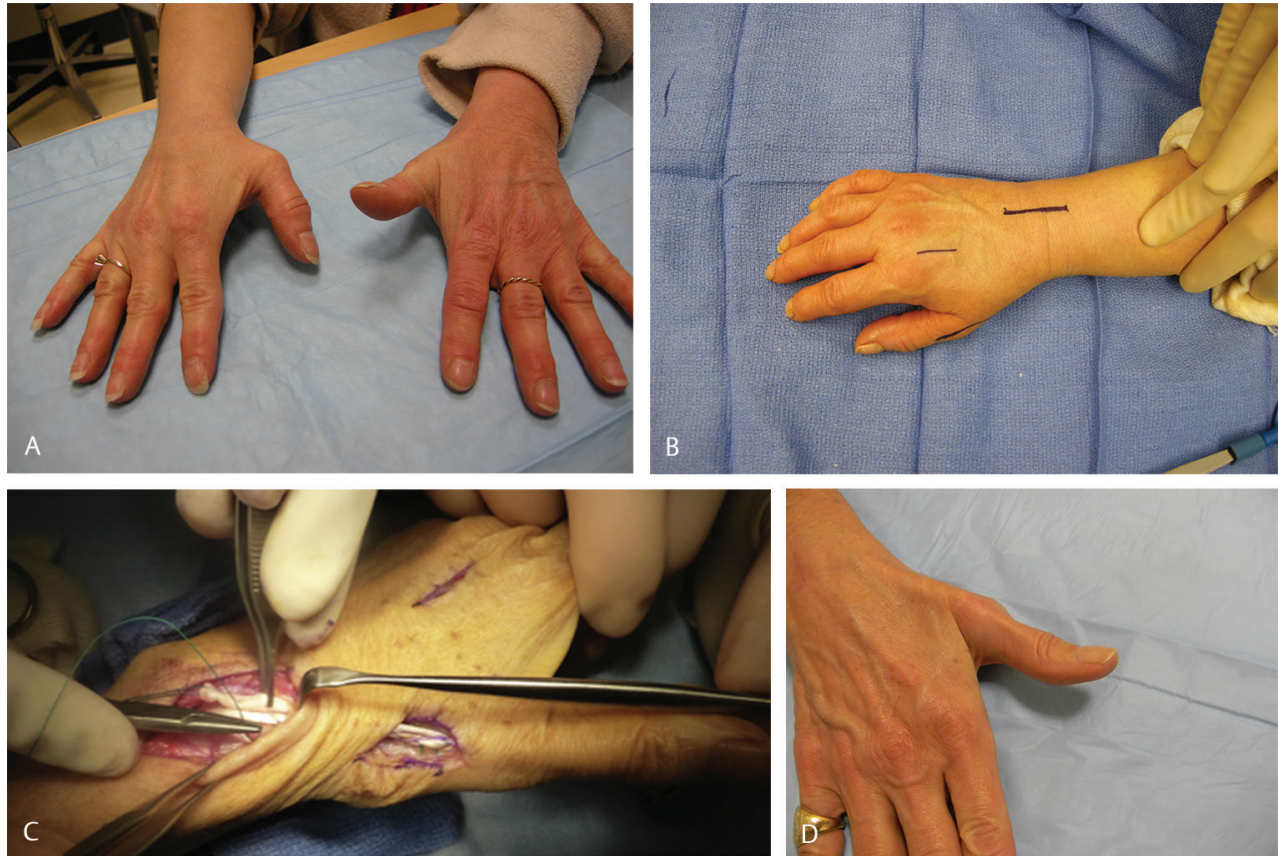

Fig. 11 (A) Spontaneous rupture of the EPL in a patient with a crack fracture of the right distal radius. (B) Incisions utilized in transferring the EIP to EPL. (C) The two-level repair of this transfer. A Pulvertaft weave is done distally and a side-to-side tenorrhaphy is done proximally. (D) Clinical recovery of complete interphalangeal extension 12 weeks after surgery. EIP, extensor indicis proprius; EPL, extensor pollicis longus

2 weeks postoperatively (-Fig. 11A-D)..$^{52}$ Patients are supported in a long opponens splint for a further 3 weeks when not performing their rehabilitative exercises. Six weeks after surgery, splinting is discarded and a strengthening program is commenced.Finally, avulsion of a tendon at the level of the musculotendinous junction is also well described and is usually not amenable to primary repair. ${ }^{5}$ In such a situation, where all digital extensors are involved, transferring the FCR 

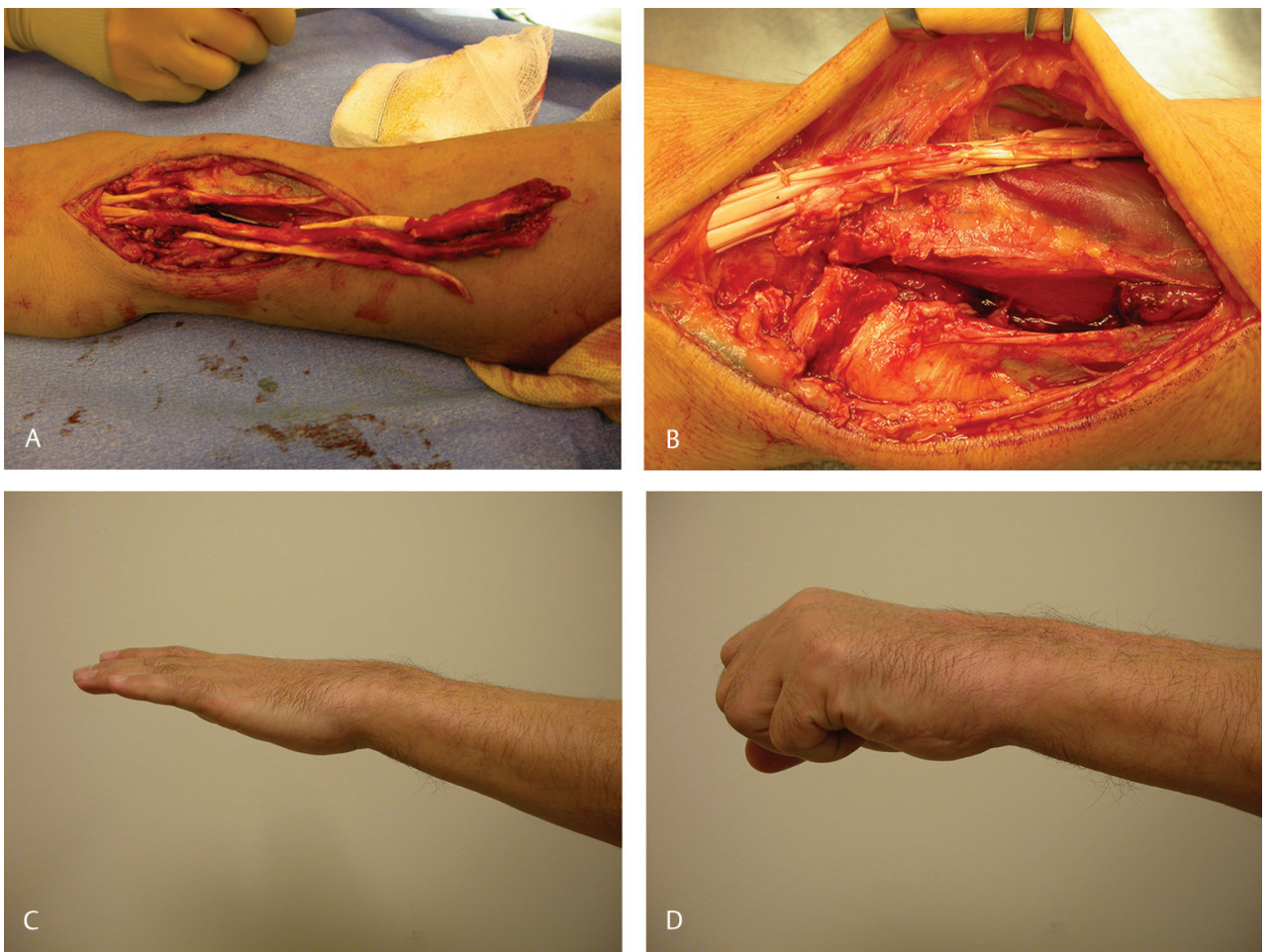

Fig. 12 (A) In this case, a 51-year-old man suffered closed traumatic rupture of the extensor digitorum communis and EIP at the musculotendinous junction after the patient's glove on his nondominant left hand became caught in a high-speed drill. (B) This was repaired with an FCR to EDC and EIP transfer. (C, D) Result at 10 weeks reveals a composite fist and excellent extension of all fingers. EDC, extensor digitorum communis; EIP, extensor indicis proprius; FCR, flexor carpi radialis.

to the digital extensors provides a very reliable transfer with a predictable outcome (-Fig. 12A-D). While situations like this are extremely uncommon, the principles that guide tendon transfers in such a situation, setting of the tension of the transfer as well as rehabilitation of the transfer, are essentially similar to the principles applied in other circumstances where digital extensors are not functioning.

\section{Conclusion}

In conclusion, tendon transfers in the upper extremity are a powerful tool that had started with polio and expanded to restore function to many patients with a variety of disease etiologies. In our experience, results from tendon transfers are usually excellent when replacing ruptured tendons or as an alternative to tendon repair, good in the treatment of peripheral nerve palsies, and not always predictable in the treatment of spastic disorders and spinal cord injury. When key principles are followed, outcomes from tendon transfers restore critical functions and dramatically improve quality of life.

\section{Conflict of Interest}

None.

\section{References}

1 Gurunluoglu R, Shafighi M, Huemer GM, Gurunluoglu A, Piza-Katzer H. Carl Nicoladoni (1847-1902): professor of surgery. Ann Surg 2004;239(2):281-292

2 Grzybowski A, Pietrzak K. Tomasz Drobnik (1858-1901) J Neurol 2016;263(3):624-625
3 Steindler A. Orthopaedic reconstruction work on hand and forearm. N Y Med J 1918;108:1117-1119

4 Bunnell J, Boyes J. Tendon transfer surgery. Orthop Clin North Am 1970;1:e54

5 Mudgal CS, Mudgal S. Closed traumatic rupture of the extensor digitorum communis and extensor indicis proprius at the musculo-tendinous junction. J Hand Surg Eur Vol 2007;32(6):675-676

6 Sammer DM, Chung KC. Tendon transfers: Part II. Transfers for ulnar nerve palsy and median nerve palsy. Plast Reconstr Surg 2009;124(3):212e-221e

7 Sammer DM, Chung KC. Tendon transfers: part I. Principles of transfer and transfers for radial nerve palsy. Plast Reconstr Surg 2009;123(5):169e-177e

8 Drijkoningen T, Peters RM, Eberlin KR. Mudgal CS, Cetrulo CL Jr. Case study: osteosynthesis, tenodesis effect, and side-to-side tendon repair in hand transplantation. Tech Hand Up Extrem Surg 2015;19(3):110-114

9 Biesalski K, Mayer L. Die physiologische sehnenverpflanzung: mit 270 zum grossen teil farbigen abbildungen. Berlin: Verlag von Julius Springer; 1916

10 Wapner KL, Taras JS, Lin SS, Chao W. Staged reconstruction for chronic rupture of both peroneal tendons using Hunter rod and flexor hallucis longus tendon transfer: a long-term followup study. Foot Ankle Int 2006;27(8):591-597

11 Horii E, An KN, Linscheid RL. Excursion of prime wrist tendons. J Hand Surg Am 1993;18(1):83-90

12 Su F-C, Chou Y-L, Yang C-S, Lin G-T, An K-N. Movement of finger joints induced by synergistic wrist motion. Clin Biomech (Bristol, Avon) 2005;20(5):491-497

13 Morris $\mathrm{CB}$. The measurement of the strength of muscle relative to the cross section. Res Q 1948;19:295-303

14 Amis A, Dowson D, Wright V. Muscle strengths and musculoskeletal geometry of the upper limb. Eng Med 1979;8:41-48 
15 Peljovich A, Ratner JA, Marino J. Update of the physiology and biomechanics of tendon transfer surgery. J Hand Surg Am 2010;35(8):1365-1369, quiz 1370

16 Lieber RL, Jacobson MD, Fazeli BM, Abrams RA, Botte MJ. Architecture of selected muscles of the arm and forearm: anatomy and implications for tendon transfer. J Hand Surg Am 1992;17(5):787-798

17 Takahashi M, Ward SR, Lieber RL. Intraoperative single-site sarcomere length measurement accurately reflects whole-muscle sarcomere length in the rabbit. J Hand Surg Am 2007;32(5):612-617

18 Fridén J, Lieber RL. Evidence for muscle attachment at relatively long lengths in tendon transfer surgery. J Hand Surg Am 1998;23(1):105-110

19 Takahashi M, Ward SR, Marchuk LL, Frank CB, Lieber RL. Asynchronous muscle and tendon adaptation after surgical tensioning procedures. J Bone Joint Surg Am 2010;92(3):664-674

20 Pulvertaft RG. Tendon grafts for flexor tendon injuries in the fingers and thumb; a study of technique and results. J Bone Joint Surg Br 1956;38-B:175-194

21 Brown SH, Hentzen ER, Kwan A, Ward SR, Fridén J, Lieber RL. Mechanical strength of the side-to-side versus Pulvertaft weave tendon repair. J Hand Surg Am 2010;35(4): 540-545

22 Rivlin M, Eberlin KR, Kachooei AR, et al. Side-to-side versus Pulvertaft extensor tenorrhaphy-a biomechanical study. J Hand Surg Am 2016;41(11):e393-e397

23 Neuhaus V, Wong G, Russo KE, Mudgal CS. Dynamic splinting with early motion following zone IV/V and TI to TIII extensor tendon repairs. J Hand Surg Am 2012;37(5):933-937

24 Rath S, Selles RW, Schreuders TA, Stam HJ, Hovius SE. A randomized clinical trial comparing immediate active motion with immobilization after tendon transfer for claw deformity. J Hand Surg Am 2009;34(3):488-494, 494.e1-494.e5

25 Gelberman RH, Botte MJ, Spiegelman JJ, Akeson WH. The excursion and deformation of repaired flexor tendons treated with protected early motion. J Hand Surg Am 1986;11(1):106-110

26 Dawson CK, Mudgal CS. Fascial augmentation tenorrhaphy for extensor tendon lacerations in the forearm. Tech Hand Up Extrem Surg 2012;16(2):72-74

27 Dabas V, Suri T, Surapuraju PK, Sural S, Dhal A. Functional restoration after early tendon transfer in high radial nerve paralysis. J Hand Surg Eur Vol 2011;36(2):135-140

28 Skoll PJ, Hudson DA, de Jager W, Singer M. Long-term results of tendon transfers for radial nerve palsy in patients with limited rehabilitation. Ann Plast Surg 2000;45(2):122-126

29 Zancolli EA. Claw-hand caused by paralysis of the intrinsic muscles: a simple surgical procedure for its correction. J Bone Joint Surg Am 1957;39-A(5):1076-1080

30 Bunnell S. Surgery of the intrinsic muscles of the hand other than those producing opposition of the thumb. J Bone Jt Surg $1942 ; 24: 1-3$

31 Hastings H II, McCollam SM. Flexor digitorum superficialis lasso tendon transfer in isolated ulnar nerve palsy: a functional evaluation. J Hand Surg Am 1994;19(2):275-280

32 Stiles H, Forrester-Brown M, Treatment of Injuries of the Spinal Peripheral Nerves. London: Frowde and Hodder \& Stoughton; 1922
33 Burkhalter WE. Restoration of power grip in ulnar nerve paralysis. Orthop Clin North Am 1974;5(2):289-303

34 Hamlin C, Littler JW. Restoration of power pinch. J Hand Surg Am 1980;5(4):396-401

35 Smith RJ. Extensor carpi radialis brevis tendon transfer for thumb adduction-a study of power pinch. J Hand Surg Am 1983;8(1):4-15

36 Brand PW. Paralytic claw hand; with special reference to paralysis in leprosy and treatment by the sublimis transfer of Stiles and Bunnell. J Bone Joint Surg Br 1958;40-B(4):618-632

37 Brand PW. Tendon grafting: Illustrated by a new operation for intrinsic paralysis of the fingers. J Bone Jt Surg 1961; 43:444-453

38 Ozkan T, Ozer K, Gülgönen A. Three tendon transfer methods in reconstruction of ulnar nerve palsy. J Hand Surg Am 2003;28(1):35-43

39 Boatright JR, Kiebzak GM. The effects of low median nerve block on thumb abduction strength. J Hand Surg Am 1997;22(5):849-852

40 Royle N. An operation for paralysis of the intrinsic muscles of the thumb. JAMA 1938;111:612-613

41 Jensen EG. Restoration of opposition of the thumb. Hand 1978;10(2):161-167

42 Camitz H. Uber die Behandlung der opposition slahmung. Acta Chir Scand 1929;65:77-81

43 Lemonas $\mathrm{P}$, Laing $\mathrm{T}$, Ghorbanian S, Malahias $\mathrm{M}$, Ragoowansi R. Extensor indicis proprius opponensplasty - the burkhalter revisited. J Hand Microsurg 2012;4(2):47-49

44 Hsiao EC, Fox IK, Tung TH, Mackinnon SE. Motor nerve transfers to restore extrinsic median nerve function: case report. Hand (N Y) 2009;4(1):92-97

45 Cooney WP, Linscheid RL, An KN. Opposition of the thumb: an anatomic and biomechanical study of tendon transfers. J Hand Surg Am 1984;9(6):777-786

46 Anderson GA, Lee V, Sundararaj GD. Extensor indicis proprius opponensplasty. J Hand Surg [Br] 1991;16(3):334-338

47 Terrono AL, Rose JH, Mulroy J, Millender LH. Camitz palmaris longus abductorplasty for severe thenar atrophy secondary to carpal tunnel syndrome. J Hand Surg Am 1993;18(2):204-206

48 Mannerfelt L, Norman O. Attrition ruptures of flexor tendons in rheumatoid arthritis caused by bony spurs in the carpal tunnel. A clinical and radiological study. J Bone Joint Surg Br 1969;51(2):270-277

49 O'Sullivan MB, Singh H, Wolf JM. Tendon transfers in the rheumatoid hand for reconstruction. Hand Clin 2016;32(3):407-416

50 Chung US, Kim JH, Seo WS, Lee KH. Tendon transfer or tendon graft for ruptured finger extensor tendons in rheumatoid hands. J Hand Surg Eur Vol 2010;35(4):279-282

51 Vaughan-Jackson OJ. Rupture of extensor tendons by attrition at the inferior radio-ulnar joint; report of two cases. J Bone Joint Surg Br 1948;30B(3):528-530

52 Sluijmer HCE, Neuhaus V, Mudgal CS. Two-level repair of the extensor indicis proprius transfer for rupture of the extensor pollicis longus. Tech Orthop 2015;30(1):43-45 